\title{
Practice Article
}

\section{A method to provide integrated care for complex medically ill patients: The INTERMED}

\author{
Cornelia H. M. Latour, RN, PhD, ${ }^{1}$ Frederik J. Huyse, $\mathrm{MD}, \mathrm{PhD},{ }^{2}$ Rien de Vos, $\mathrm{PhD}^{3}$ and \\ Wilhelmus A. B. Stalman, MD, $\mathrm{PhD}^{4}$ \\ ${ }^{1}$ Psychiatric Consultation and Liaison Service, ${ }^{4}$ Department of General Practice, Institute for Research in Extramural \\ Medicine, Vrije University Medical Center, ${ }^{2}$ Department of General Internal Medicine, University Medical Center Groningen, \\ Groningen and ${ }^{3}$ Department of Clinical Epidemiology and Biostatistics, Academic Medical Center, University of Amsterdam, \\ Amsterdam, the Netherlands
}

\begin{abstract}
A growing number of nursing subspecializations have been developed in recent decades. Topics of concern are that care is not tailored to cope with the growing number of patients with more than one chronic disease, there is an increase in co-ordination problems in the care that is provided for this group of complex patients, and the care for these complex patients is extremely fragmented. The assessment of health risks resulting in coordinated care with effective communication is vital for multimorbid patients. Our aim is to describe a systematic approach (the INTERMED [IM] method) to identify complex patients who are in need of integrated care and its applicability to the nursing process. We demonstrate the ability of the IM method to quantify, weigh, and classify the complexity of problems. The method is applied in Europe and will be started in the USA in a pharmacy program. The IM is presented as a decision-support system for multidisciplinary teams with nurse co-ordinators.
\end{abstract}

Key words

case management, complexity, integrated care, INTERMED, nursing process.

\section{INTRODUCTION}

Despite all the medical progress that has been achieved, for a growing number of complex patients, our current health care is in need of adjustments. Health care suffers from fragmentation and a related lack of communication between health-care providers. The problem is not unique to the European health-care system because the situation is similar in the USA. This leads to poorly developed health-care processes and a restricted scope of communication with patients (Institute of Medicine, 2001). Complex patients can be found with acute or chronic medical, neurological, obstetrical or surgical condition(s), including patients with unexplained physical conditions, and psychiatric comorbidity and psychiatric disorders, which are the direct consequence of primary medical conditions, such as organic psychiatric disorders and substance abuse (Gitlin et al., 2004). Examples are confused orthopedic patients, diabetic patients with a depressive disorder, and HIV patients with a drug addiction. When not addressed appropriately, the combination of psychiatric and physical comorbidities has a deleterious effect on health outcomes and the utilization of health-care resources (Fink,

Correspondence address: F. Huyse, Department of General Internal Medicine, University Medical Centre Groningen, Hanzeplein 1, 9700RB Groningen, The Netherlands. Email: f.j.huijse@int.umcg.nl

Received 23 April 2006; accepted 25 October 2006
1990; Saravay \& Lavin, 1994; Saravay et al., 1996; Lustman et al., 2000; Ludman et al., 2004; Gilmer et al., 2005).

Based on a patient case description, the aim of this article is to describe an approach (the INTERMED [IM] method) to identify complex patients who are in need of integrated care and its applicability in relation to the nursing process. The IM method can be seen as a decision-support system.

\section{DECISION-SUPPORT SYSTEMS}

A decision-support system (DSS) can be defined as a system that is:

... designed to aid directly in clinical decision-making, in which characteristics of individual patients are used to generate patient-specific assessments or recommendations that are then presented to clinicians for considerations (Hunt et al., 1998: 1339).

A systematic review identified 11 studies in which the features most crucial for improving clinical practice in clinical DSSs are summarized. They conclude that DSSs do improve clinical practice (Kawamoto et al., 2005). The identified studies direct proceedings for a specific part of care. For instance, one study reported that computer-generated chart reminders for mammography referral, plus mailed patient reminders, were superior to computer-generated chart reminders for mammography referral only (Burack et al., 1996). 


\section{INTERMED METHOD}

In contrast to DSSs with a focus on a single condition, the IM is a DSS that assesses multiple health risks and health needs by means of a semistructured interview based on the biopsychosocial model (Engel, 1977; Huyse et al., 1999; Stiefel et al., 1999b; de Jonge et al., 2001; Stiefel et al., 2006; Appendix I). The method has been developed to facilitate the care of patients with multiple conditions (comorbidities). The more conditions a patient has, the more complex a patient is, as well as the related need for integrated care (de Jonge et al., $2005 ; 2006)$. The IM is based on clinical experience with life charts and epidemiological literature on the characteristics influencing patients' treatment responses and health-care use (Stiefel et al., 2006). The method is based on an interview completed by a health-care worker, usually a nurse and preferably a member of a regular staff, as it is important that the patient has the opportunity to make contact with the nurse. When the patient is too sick or too confused, information is collected from family members or others involved in the treatment of the patient. All available information should be collected before starting the interview, in order to avoid redundant interviewing. Depending on the type of organization or department, an interview is done for all patients (for instance, preoperatively for high-risk operations i.e. department for liver transplantation) or for a specific set of patients (for instance, in an internal medicine department only for the elderly, patients with more than two comorbidities or a psychiatric disease in the [recent] history) (Huyse et al., 2006).

The collected data are organized in a matrix. The matrix has four dimensions: potential biological, psychological, and social health risks or needs, and the patient's relationship with the health-care system. These are fitted on a time-axis: history, current status, and prognoses (Table 1). Each dimension consists of five variables, each rated on a 4-point scale, ranging from $0-3$. The matrix contains 20 variables (16 risks and four prognoses). The format of each variable is the same and is based on a theory to enhance communication (Lyons, 2006). The generic answering structure is: $0=$ no vulnerability, no action needed; 1 = a mild vulnerability, watchful waiting; 2 = a moderate vulnerability, action needed; and $3=a$ severe vulnerability, direct or intensive action needed (Table 2). Consequently, the maximum score is 60 points. The severity of the risks can be visualized $(0=$ green, $1=$ yellow, $2=$ orange, and $3=$ red). In contrast to lengthy psychometric instruments, which have primarily been developed for research and not for clinical care, in communimetric instruments, only one variable is used to assess one risk. This, of course, increases its clinical applicability. However, the reliability of these single assessments becomes crucial. Therefore, all the scoring options for each variable are clearly specified in a manual with defined clinical anchor points (INTERMED Foundation, 2006). The method has been extensively evaluated (Stiefel et al., 2006).

Validation studies have been performed in several populations. There is a high correlation with the indicators for case and care complexity in several somatic patient populations (Stiefel et al., 1999a; Fischer et al., 2000; Koch et al., 2001; Di Gangi Herms et al., 2003; de Jonge et al., 2003a). In addition, we were able to establish a (preliminary) cut-off point of $\geq 21$. It was found to detect patients at risk of poor discharge health status: as such, we consider these patients as complex, indicating the need for some form of case management (de Jonge et al., 2003a).

The inter-rater reliability of the IM was assessed by calculating the agreement of two independent raters, based on the same information. The correlations between the total scores

Table 1. INTERMED vignette

\begin{tabular}{|c|c|c|c|}
\hline Dimension & History & Current situation & Prognoses \\
\hline \multirow[t]{2}{*}{ Biological } & Chronicity & Severity of symptoms & \multirow[t]{2}{*}{ Complications and life threat } \\
\hline & Diagnostic dilemma & Diagnostic challenge & \\
\hline \multirow[t]{2}{*}{ Psychological } & Restrictions in coping & Resistance to treatment & \multirow[t]{2}{*}{ Mental health threat } \\
\hline & Psychiatric dysfunctioning & Psychiatric symptoms & \\
\hline \multirow[t]{2}{*}{ Social } & Restrictions in integration & Residential instability & \multirow[t]{2}{*}{ Social vulnerability } \\
\hline & Social dysfunctioning & Restrictions of network & \\
\hline \multirow[t]{2}{*}{ Health care } & Intensity of treatment & Organization of care & \multirow[t]{2}{*}{ Co-ordination } \\
\hline & Treatment experience & Appropriateness of referral & \\
\hline
\end{tabular}

Reproduced from Huyse et al. (1999), with permission from Elsevier.

Table 2. Score labels for INTERMED

\begin{tabular}{llll}
\hline Numerical & Visual & \multicolumn{1}{c}{ Score } & \multicolumn{1}{c}{ Action } \\
\hline 3 & Red & Severe vulnerability or care needs & Immediate and/or intensive treatment \\
2 & Orange & Moderate vulnerability or care needs & Treatment \\
1 & Yellow & Mild vulnerability or care needs & Monitoring or preventive intervention \\
0 & Green & No vulnerability or care needs & No action needed \\
\hline
\end{tabular}


of the two raters ranged from $0.91-0.96$. At item level, in $83 \%$ of the assessments, there were no differences between the raters, in $16 \%$ there was a one-point difference, and in $1 \%$ there was a two-point difference. Based on a cut-off score of 20/21, a ê of 0.85 was found (de Jonge et al., 2002).

The virtue of the IM is its systematic approach to the patient and its ability to quantify, weigh, and classify the complexity of problems by assessing the patient's vulnerabilities in terms of health risks and needs (Fig. 1). As such, it is presented as a DSS, facilitated by visualization of the risks in a grid, showing the need for action in the colors of traffic lights. There are indications that patients whose treatment plan has been formulated according to the IM method experience a better quality of life and have a shorter stay in hospital (de Jonge et al., 2003b). However, there are also indications that if the case manager (CM) makes patients more aware of their impaired functioning and vulnerabilities by discussing these aspects extensively as part of the intervention, this demands more intense short-term care and, therefore, results in an increase in health-care costs (Frasure-Smith et al., 1997; Latour et al., 2006).

A computer program was developed in order to score the patient and to systematically document the received information. A web-based service will soon become available and a course will be offered in order to learn how to work with this method. Currently, the method is applied in clinical practice in two university hospitals in the Netherlands, in Switzerland, Germany, and Sweden, and will be started in the USA in a pharmacy program.

\section{PATIENT CASE DESCRIPTION}

A 27 year old woman is admitted to a gastroenterology ward for the evaluation of diarrhea. Her condition has deteriorated as she has lost $\approx 10$ kilos in the past month and she has informed her doctor that she is almost incapable of doing anything at home. As a result, he admits the patient immediately. Although extensive diagnostic evaluations had been performed in the past and an enteritis regionalis is most likely, a definitive diagnosis still has to be established. The patient has also been suffering from systemic lupus erythematosis (SLE) for the past 4 years, with the kidneys as a focus of primary expression. Therefore, she is being treated by a nephrologist. A relationship between the diarrhea and the SLE is a diagnostic option.

During the admission process, the nurse obtains additional information. The patient tells her that, initially, $\approx 4$ years ago, the complaints were vague. Marital stress had once been

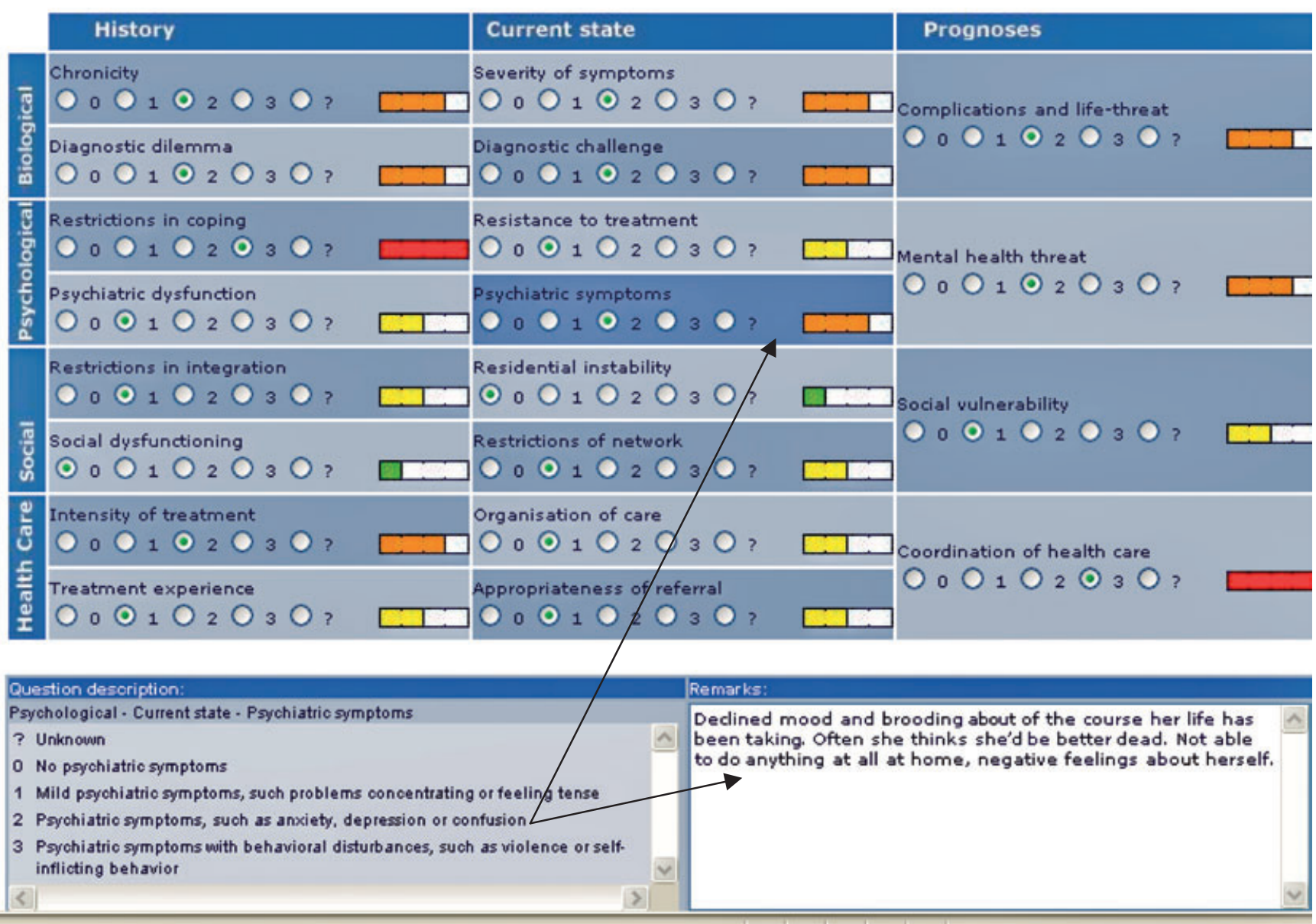

Figure 1. Visualization of the patients' vulnerabilities in terms of health risks and needs. 
suggested by her family physician, who thought that she might have chronic fatigue syndrome. The patient felt offended by this suggestion and therefore lost all confidence in doctors. The family physician eventually referred her to an internist, who arranged for her to undergo many diagnostic tests before diagnosing SLE with primary expression in the kidneys. The nephrologists then initiated treatment with corticosteroids and cyclofosfamide. Although the doctors were satisfied with the results and she felt a little better, she hated the congested look of her face that was induced by the medication. She therefore stopped taking her medication sometimes. The patient now shows resistance and has doubts about her therapy, but basically does what the doctors tell her to do. After a recent visit to an infertility clinic, it also became clear that, in view of her current illness, her chances of becoming pregnant were considered to be minimal and that she would not be eligible to participate in a fertilization program. From being a cheerful adolescent, she has gradually become a person with negative feelings about herself, feelings of not being a good partner for her husband, and always feeling tired. As a result, she has neglected her leisure activities. About 1 year ago, after being informed that she would not be able to have children, her mental condition declined and she took an overdose of her medication. As she did not tell anyone about this suicide attempt, she received no specific treatment. The relationship with her husband also deteriorated during this period, but it has now stabilized and her husband is supportive. Although her relatives live far away, she speaks to them regularly, and she also has some good friends. She managed to get a volunteer job in a day-care center for small children, but during the past month, she has been unable to cope. The diarrhea makes her feel weak. Her mood has deteriorated again and she has been thinking about the way her life has changed. Again, she often wishes that she was dead.

\section{Classification and scoring with the INTERMED}

When described according to the four dimensions of the IM, we come to an interpretation of the history and current status presented in this case description. The classification between brackets refers to the specific IM variable, ( ), and its actual scoring, [ ] (Tables 1,2).

\section{Biological dimension}

This patient suffers from a complicated medical illness (chronicity [2]), but definitive diagnoses still have not been established (diagnostic dilemma [2]). The patient's already complicated medical condition has become even more complex due to new symptoms of unclear origin (diagnostic challenge [2]), which make her almost incapable of doing anything at home (severity of symptoms [2]).

\section{Psychological dimension}

From a cheerful adolescent, she has become a person with negative self-esteem (psychiatric dysfunction [1]). She has a history of attempted suicide (restrictions in coping [3]), and at the present moment she often thinks that it would be better if she was dead. This suggests that the patient suffers from a depressive disorder (psychiatric symptoms [2]). Doubts about the therapy and treatment in the past and her current psychological state make her vulnerable for non-compliance (resistance to treatment [1]).

\section{Social dimension}

The patient has a supportive husband and good contact with her family and friends, but no work (restrictions of network [1]). She is able to maintain meaningful relationships with others (social dysfunction [0]), but she has been unable to continue with her volunteer work for the past month and has neglected her leisure activities for a long time (restrictions in integration [1]). Although she feels too ill to do anything at home, with some help from her husband, she is able to live independently at home (residential instability [0]).

\section{Organization of health care dimension}

Various specialists have been involved in the treatment of this patient in the past 5 years, but there has been no hospital admission (intensity of treatment [2]). Her lack of confidence in doctors has been influenced through her history of changing diagnoses and she felt offended by the suggestion that she suffered from chronic fatigue syndrome (treatment experience [1]). At this moment, several specialists are involved (organization of care [1]). Her current hospital admission was unplanned (appropriateness of referral [1]).

The four dimensions of the IM prognosis are as follows:

\section{Biological dimension}

The complexity of this patient is reflected in a high subscore (10 points) on the biological axis. The prognosis for this patient is a substantial limitation in her activities in daily life related to her chronic condition and related need for physical care (complications and life threat [2]).

\section{Psychological dimension}

A psychiatric consultation should confirm the suspicion of depression (mental health threat [2]) and a (psychiatric) liaison nurse should instruct the ward staff with regard to the approach towards the patient and arrange for postdischarge psychiatric care. Action is needed in order to increase the patient's compliance. This is a psychologically vulnerable patient, as is indicated by a high subscore of nine points on the variables of the psychological axis.

\section{Social dimension}

Despite her vulnerabilities on the other axes, this patient has reasonably good social circumstances, reflected in a low subscore of three points on the social axis. It is expected that after her discharge from hospital, she will be able to do some housekeeping again. She will need support to return to her 
volunteer work and to recommence her leisure activities (social vulnerability [1]).

\section{Organization of health care dimension}

Serious efforts are needed to organize the care (co-ordination [3]) for this patient, as is reflected in a high total score of 30 points, 11 points above the cut-off score, indicating the need for case management (de Jonge et al., 2003a).

\section{From classification to co-ordination of care}

The IM method facilitates the correct identification of risks, but might only be effective when followed by an integrated care plan. This care plan should include state-of-the-art medical insights, a multidisciplinary approach, and co-ordination of all the health-care disciplines that are involved. More specifically in addition to the physical cave, the following initiatives should occur:

\section{Psychiatric consultation}

The loss of vitality can be seen as a consequence of the illness, the infertility, the depression or the lack of a meaningful working life. Therefore, a medical examination and analyses must be completed by a psychiatric consultant. If the patient is depressed, therapy has to be started during hospitalization. This can include medication, psychotherapy, and psychoeducation. The ward staff has to be instructed by a (preferably psychiatric) nurse with regard to their approach towards the patient. Depending on the local policies, after hospitalization, the ambulatory CM or a psychiatrist will treat the patient with medication and psychotherapy (Unutzer et al., 2002).

\section{Multidisciplinary case conference}

A multidisciplinary case conference during the hospital stay will be organized as soon as a psychiatric consultation has occurred. The co-ordination of care is necessary when the patient is discharged, so the ambulatory $\mathrm{CM}$ will be invited to attend this case conference. One of the goals during the case conference is the organization of the co-ordinated care, especially because this patient has a history of changing diagnoses and a lack of confidence in doctors. For the period of hospitalization, a care co-ordinator (usually a nurse) will be assigned. Three months after discharge, an evaluation will be organized by the ambulatory CM in order to assess whether the goals have been achieved or should be adjusted.

\section{Ambulatory case manager}

The CM should, in general, concentrate on the following: interventions focusing on the complex diagnostic problems, such as the risk of rare diseases, interventions focusing on the treatment or the consequences of a psychiatric disease, and a disease management part focusing on the complications of the various physical diseases, including rehabilitation and interventions focusing on the effects of physical dependency. Also, the CM will focus on the prevention of communication problems between the patient and the care-providers due to the complexity of the problems, as well as non-compliance, which might be the result from these complexities or the psychiatric complaint (depression). Finally, there is a focus on interventions concerning the consequences of social restrictions (Huyse et al., 2001a; Unutzer et al., 2002; Harpol et al., 2005). The ambulatory CM will visit the patient on a regular basis at home after discharge and should be easily accessible for assistance.

\section{Care co-ordinator}

One goal of the care co-ordinator during the hospital stay is to carry out and oversee the multidisciplinary treatment plan and to communicate with the patient and her husband to ensure that they are well-informed and unambiguous about the diagnoses and the treatment plan. Another goal is to improve postdischarge compliance, so during the patient's hospital stay, the care co-ordinator has to negotiate with the patient about the medication regime and further lifestyle advice must be discussed.

\section{Stable staff}

As this is a chronic case with a complex medical history and related communication problems, the same staff members preferably should treat the patient.

\section{Outcomes (during hospital stay)}

An enteritis regionalis was diagnosed during the hospital stay and the patient was treated successfully. The psychiatrist diagnosed depression and treatment started (medication and psychotherapy). The ward staff was instructed by a psychiatric nurse and, with a structured revalidation plan, the ward succeeded in motivating the patient with exercizing, medication regime, and intake of food.

\section{Outcomes (after discharge)}

The ambulatory CM, under the supervision of a psychiatrist, continued the treatment for depression. The CM controlled for the symptoms of rare diseases and referred the patient once to the nephrologists, but she was all right.

The CM began to visit the patient every three weeks, discussing disease symptoms, compliance, lifestyle, and communication aspects with the patient and other involved health-care professionals. Continuity of involved health-care professionals was achieved. Under the supervision of a psychiatrist, the CM continued the patient's psychological treatment for depression. Based on the symptoms reported, and extra visit to the nephrologists was planned. In addition, the patient consulted the CM by telephone during the next three months; twice regarding uncertainties about the prescribed medication. As her condition improved after six weeks, the $\mathrm{CM}$ supported the patient in making an appointment with the day-care centre in order to restart her volunteer work. As she was too weak to start fulltime, the patient volunteered three times per week for a maximum of 1.5 hours per day. 


\section{DISCUSSION}

The aim of this article was to describe a systematic approach (the IM method) to identify complex patients and its applicability in relation to the nursing process.

The development of medical and nursing science in recent decades has resulted in a growing number of medical and nursing subspecializations in an attempt to organize health care for patients with complex chronic diseases in the most efficient way. As a result, a variety of disease management programs have been developed (West et al., 1997; Aubert et al., 1998; Harris et al., 1998; Stewart et al., 1999). Disease management is defined as a population-based approach to chronic diseases (Weingarten et al., 2002). For example, diabetes or heart failure are diseases for which disease management programs are suitable. However, although these programs are an important step towards providing integrated care for patients, they are not tailored to cope with multimorbid patients and often exclude psychiatric health care (Huyse et al., 2006). Additional morbidity, including psychiatric disorders and inherent behavioral aspects, such as non-compliance, are factors that interfere with the efficient utilization of care facilities, quality care management and quality of life (Kathol et al., 2005; Huyse et al., 2006). Moreover, in addition to patients with chronic diseases, there are highly complex patients who should be identified and treated appropriately.

Changes are required if we are to meet the care needs of these complex and vulnerable patients (Institute of Medicine, 2001) With a considerable increase in the number of such patients, we need to provide more productive care for these patients, guided by DSSs, such as the IM method.

The IM gives a quick and concise overview of the health risks or needs of a patient. However, it is important to emphasize that the IM is not a diagnostic instrument, so it does not lead to medical or nursing diagnoses. The IM describes the domains of the patient but, even more importantly, it describes in particular the interface between the domains. The IM is designed as an instrument to facilitate interdisciplinary communication, to be used by workers in various health-care disciplines with different levels of education. It offers a framework for a treatment plan, including who should be involved in providing the care. The IM describes the extent to which the co-ordination of care is needed, but it does not describe the type of casemanagement interventions that are needed or exactly how the care should be organized.

The applicability and effectiveness of the IM has been assessed in different care settings (an internal medicine ward, in an ambulatory setting, and in a rehabilitation center for traffic accidents) and is not dependent on the type of organization or even the health-care system of a country. This gives many opportunities for the implementation of the IM. However, most studies have investigated the implementation of the IM among hospital patients, and more research on the IM as a longitudinal instrument is needed, especially in a primary health-care setting.

From our own experience, we know that co-ordinated care for outpatients is more difficult to establish than for inpatients (de Jonge et al., 2003b). Many different health-care organizations and health-care workers can be involved in providing care for complex outpatients (e.g. family physicians, specialists, allied health-care professionals, district nurses, psychiatric health-care workers). We found that communication about inpatients among the members of the treatment team was more effective as the communication lines were short, the case conferences were arranged more quickly, and informal contact was possible. For instance, in a neurology ward, the implementation of the IM method resulted in reducing the time needed for the weekly multidisciplinary conference from $90 \mathrm{~min}$ to $45 \mathrm{~min}$.

It is clear that the IM cannot stand alone. It requires a change in focus from disease-oriented care towards integrated care. It requires training, time, effort, and often specialist expertise, such as psychiatric nursing skills (Happell \& Platania-Phung, 2005), and the use of a change model to guide implementation is essential (Cohen et al., 2005). It also requires the approval of the organization and the purpose of the integrated program must be clear to all those who are involved. A nurse with a broad range of competence, with experience in general health care, as well as psychiatric health care, should be appointed to fulfill the role of CM.

In light of the above, a vision for integrated care needs to be shared by health-care workers, policy-makers, and politicians. We need leaders who are able to implement integrated care programs. Health-care workers need to be involved in integrated care programs, and they need commitment and support from management. Finances and resources should be available in order to realize the implementation of integrated care programs, and there is a need to develop a culture of quality improvement (Institute of Medicine, 2001).

Measuring complexity, for instance, by means of the IM, should be a basic principle of health care. Integrated care programs should be provided where health-care workers deal with complex, medically ill patients and when patients and health-care workers experience communication problems, organizational problems or tendencies of avoidance or denial. Future research should link up with already existing care programs. Follow-up should be of adequate duration as there are indications that integrated care programs are only (cost) effective in the long term, not in the short term.

In our opinion, decision-support methods, such as the IM, focusing on complexity, will eventually result in more efficient and better quality care.

\section{REFERENCES}

Aubert RE, Herman WH, Waters J et al. Nurse case management to improve glycemic control in diabetic patients in a health maintenance organization. A randomized, controlled trial. Ann. Intern. Med. 1998; 129: 605-612.

Burack RC, Gimotty PA, George J, Simon MS, Dews P, Moncrease A. The effect of patient and physician reminders on use of screening mammography in a health maintenance organization. Results of a randomized controlled trial. Cancer 1996; 78: 1708 1721.

Cohen DJ, Tallia AF, Crabtree BF, Young DM. Implementing health behavior change in primary care: lessons from prescription for health. Ann. Fam. Med. 2005; 3 (Suppl. 2): 12-19. 
Di Gangi Herms AM, de Pinggera GMJP, Strasser H, Sollner W. Assessing health care needs and clinical outcome with urological case complexity: a study using INTERMED. Psychosomatics 2003; 44: 196-203.

Engel GL. The need for a new medical model: a challenge for biomedicine. Science 1977; 196: 129-136.

Fink P. Mental illness and admission to general hospitals: a register investigation. Acta Psychiatr. Scand. 1990; 82: 458-462.

Fischer CJ, Stiefel FC, de Jonge P et al. Case complexity and clinical outcome in diabetes mellitus. A prospective study using the INTERMED. Diabetes Metab. 2000; 26: 295-302.

Frasure-Smith N, Lesperance F, Prince RH et al. Randomised trial of home-based psychosocial nursing intervention for patients recovering from myocardial infarction. Lancet 1997; 350: 473479.

Gilmer TP, O'Connor PJ, Rush WA et al. Predictors of health care costs in adults with diabetes. Diabetes Care 2005; 28: 59-64.

Gitlin DF, Levenson JL, Lyketsos CG. Psychosomatic medicine: a new psychiatric subspecialty. Acad. Psychiatry 2004; 28: 4-11.

Happell B, Platania-Phung C. Mental health issues within the general health care system: the challenge for nursing education in Australia. Nurs. Educ. Today 2005; 25: 465-471.

Harpole LH, Williams JW, Olsen MK et al. Improving depression outcomes in older adults with co-morbid medical illness. Gen. Hosp. Psychiatry 2005; 27: 4-12.

Harris LE, Luft FC, Rudy DW, Kesterson JG, Tierney WM. Effects of multidisciplinary case management in patients with chronic renal insufficiency. Am. J. Med. 1998; 105: 464-471.

Hunt DL, Haynes RB, Hanna SE et al. Effects of computer-based clinical decision support systems on physician performance and patient outcomes: a systematic review. JAMA 1998; 280: 13391346.

Huyse FJ, Lyons JS, Stiefel FC et al. "INTERMED": a method to assess health service needs. I. Development and reliability. Gen. Hosp. Psychiatry 1999; 21: 39-48.

Huyse FJ, Lyons JS, Stiefel F, Slaets J, de Jonge P, Latour C. Operationalizing the biopsychosocial model: the INTERMED. Psychosomatics 2001a; 42: 5-13.

Huyse FJ, de Jonge P, Slaets JP, Herzog T et al. COMPRI - an instrument to detect patients with complex care needs: results from a European study. Psychosomatics 2001b; 42: 222-228.

Huyse FJ, Stiefel FC, de Jonge P. Identifiers, or "red flags," of complexity and need for integrated care. Med. Clin. North Am. 2006; 90: 703-712.

Institute of Medicine. Crossing the Quality Chasm: A New Health System for the 21st Century. Washington: The National Academies Press, 2001.

INTERMED Foundation. INTERMED. 2006. [Cited 1 Dec 2006.] Available from URL: http://www.INTERMEDfoundation.org.

de Jonge P, Bauer I, Huyse FJ, Latour $\mathrm{CH}$. Medical inpatients at risk of extended hospital stay and poor discharge health status: detection with COMPRI and INTERMED. Psychosom. Med. 2003a; 65: 534-541.

de Jonge P, Huyse FJ, Slaets JP, Sollner W, Stiefel FC. Operationalization of biopsychosocial case complexity in general health care: the INTERMED project. Aust. N. Z. J. Psychiatry 2005; 39: 795-799.

de Jonge P, Huyse FJ, Stiefel FC. Case and care complexity in the medically ill. Med. Clin. North Am. 2006; 90: 679-692. de Jonge P, Huyse FJ, Stiefel FC, Slaets JP, Gans RO. INTERMED - a clinical instrument for biopsychosocial assessment. Psychosomatics 2001; 42: 106-109.

de Jonge $\mathrm{P}$, Latour $\mathrm{C}$, Huyse FJ. Interrater reliability of the INTERMED in a heterogeneous somatic population. J. Psychosom. Res. 2002; 52: 25-27.

de Jonge P, Latour CH, Huyse FJ. Implementing psychiatric interventions on a medical ward: effects on patients' quality of life and length of hospital stay. Psychosom. Med. 2003b; 65: 997-1002.

Kathol RG, McAlpine D, Kishi Y et al. General medical and pharmacy claims expenditures in users of behavioral health services. $J$. Gen. Intern. Med. 2005; 20: 160-167.

Kawamoto K, Houlihan CA, Balas EA et al. Improving clinical practice using clinical decision support systems: a systematic review of trials to identify features critical to success. BMJ 2005; 330: 765773.

Koch N, Stiefel F, de Jonge P et al. Identification of case complexity and increased health care utilization in patients with rheumatoid arthritis. Arthr. Rheumatol. 2001; 45: 216-221.

Latour CHM, de Vos R, Huyse FJ, de Jonge P, van Gemert EAM, Stalman WAB. Effectiveness of post-discharge case management in general medical outpatients: a randomized controlled trial. Psychosomatics 2006; 47: 421-429.

Ludman EJ, Katon W, Russo J et al. Depression and diabetes symptom burden. Gen. Hosp. Psychiatry 2004; 26: 430-436.

Lustman PJ, Anderson RJ, Freedland KE, Carney RM, Clouse RE. Depression and poor glycemic control: a meta-analytic review of the literature. Diabetes Care 2000; 23: 934-942.

Lyons JS. The complexity of communication in an environment with multiple disciplines and professionals: communimetrics and decision support. Med. Clin. North. Am. 2006; 90: 693-701.

Saravay SM, Lavin M. Psychiatric comorbidity and length of stay in the general hospital. A critical review of outcome studies. Psychosomatics 1994; 35: 233-252.

Saravay SM, Pollack S, Steinberg MD, Weinschel B, Habert M. Fouryear follow-up of the influence of psychological comorbidity on medical rehospitalization. Am. J. Psychiatry 1996; 153: 397-403.

Stewart S, Marley JE, Horowitz JD. Effects of a multidisciplinary, home-based intervention on unplanned readmissions and survival among patients with chronic congestive heart failure: a randomised controlled study. Lancet 1999; 354: 1077-1083.

Stiefel FC, de Jonge P, Huyse FJ, Slaets JP et al. INTERMED - an assessment and classification system for case complexity. Results in patients with low back pain. Spine 1999a; 24: 378-384.

Stiefel FC, Huyse FJ, Sollner W et al. Operationalizing integrated care on a clinical level: the INTERMED project. Med. Clin. North Am. 2006; 90: 713-758.

Stiefel FC, de Jonge P, Huyse FJ et al. "INTERMED": a method to assess health service needs. II. Results on its validity and clinical use. Gen. Hosp. Psychiatry 1999b; 21: 49-56.

Unutzer J, Katon W, Callahan CM et al. Collaborative care management of late-life depression in the primary care setting: a randomized controlled trial. JAMA 2002; 288: 2836-2845.

Weingarten SR, Henning JM, Badamgarav E et al. Interventions used in disease management programmes for patients with chronic illness-which ones work? Meta-analysis of published reports. BMJ 2002; 325: 925.

West JA, Miller NH, Parker KM et al. A comprehensive management system for heart failure improves clinical outcomes and reduces medical resource utilization. Am. J. Cardiol. 1997; 79: 58-63. 


\section{APPENDIX I}

\section{Leading questions of the INTERMED interview}

-Now, first of all, I would like to better understand how you feel physically?

(Biological, current situation, severity of symptoms)

-I will tell you what I know about the reason for your admission and your current state. You should correct me when I am wrong.

(Biological, current situation, severity of symptoms, diagnostic challenge and health care, current situation, appropriateness of referral)

-Now, I would like to know how you felt emotionally during the last week?

(Psychological, current situation, psychiatric symptoms)

-I would like to have some more information concerning physical illnesses and treatments in the past 5 years.

(Biological, history, chronicity, diagnostic dilemma)

-Who have been the doctors who have been taking care of you in the last 5 years?

(Health care, history, intensity of treatment)

-Have you ever seen a psychiatrist in your life or have there been periods that you have been anxious, depressed or confused?

(Psychological, history, psychiatric dysfunctioning)

-Now, who are the doctors, nurses, social workers or psychologists who you are currently seeing and who take care of you?
(Health care, current situation, organization of care)

-Have there been issues with doctors during the last 5 years that gave you a bad feeling to such an extent that it might interfere with your trust in doctors?

(Health care, history, treatment experience)

-I would like to know how you follow your doctor's recommendations? Are you a person who is, generally speaking, inclined to do what doctors say?

(Psychological, current situation, resistance to treatment)

-Now, I would like to change the subject and ask you how you currently live?

(Social, history, restrictions in integration and social situation, current situation, residential instability)

-Now, I would like to know what kind of person you are. Generally speaking, are you an easy-going person?

(Social, social history, social dysfunctioning and current situation, restrictions in network)

-Now, coming to the end of the interview, I would like to ask you about your smoking and drinking habits and their relation to the current problems.

(Psychological, history, restrictions in coping)

-Do you think we missed any pertinent information?

-I finally would like to know how you have experienced this interview? Do you think that this will be helpful information or did you think this was inappropriate? 